\title{
Study on Environment Perception and Automatic Navigation Technology for the Automatic Parking System
}

\author{
Yibing Zhao' ${ }^{1}$, Jining Li $^{2}$, Yuan Yang1* \\ ${ }^{1}$ Department of Vehicle Engineering, State Key Laboratory of Structural Analysis for Industrial Equipment, \\ Dalian University of Technology, Dalian, China \\ ${ }^{2}$ The Digital Arts Department, Dalian Neusoft Institute of Information, Dalian, China \\ Email: zhaoyibing005@163.com
}

Received 26 June 2015; accepted 15 October 2015; published 22 October 2015

\section{Abstract}

The traffic accidents, traffic jams and parking problems come to appear, and have caused more and more concerns of people. The automatic parking system can help us a lot to solve these problems. The automatic parking system in this paper includes two sections, environment perception and automatic controller. The median filter and least square method are used to process the laser sensor data. Then, feasible parking space can be displayed by using K-means clustering method. The Matlab fuzzy GUI is used to establish the fuzzy controller. The kinematics equation of car is utilized to simulate the automatic vertical parking in the Matlab/simulation with the different initial path angles. Experiment results show that the environmental perception method has perfect performance and the controlling algorithm of the automatic parking system has good feasibility.

\section{Keywords}

\section{Environment Perception, Automatic Navigation, Automatic Parking System}

\section{Introduction}

With the increase of the cars, most cities are facing a series of problems such as the traffic jams, parking difficulties and the imperfect road traffic management facilities [1]. According to statistics, the accidents caused by parking account for about 30\% in China and 20\% in America. The traffic administrative departments have advised owners to install multi-curvature rear-view mirror to reduce the blind areas and improved the vehicle's safety performance. In order to ensure the safety of the parking, most automobile manufacturers have studied many parking assist systems.

In 2007, the Lexus first equipped intelligent Parking Assist System APGS (Advanced Parking Guidance System) in LS460L. The system can help the drivers complete parallel and vertical parking successfully. As the new ESO and Tiguan entered China in 2011, the Volkswagen's second generation automatic parking assist system

\footnotetext{
"Corresponding author.
}

How to cite this paper: Zhao, Y.B., Li, J.N. and Yang, Y. (2015) Study on Environment Perception and Automatic Navigation Technology for the Automatic Parking System. World Journal of Engineering and Technology, 3, 46-51. 
use the ultrasonic sensor to detect the parking space by scanning road on both sides and prompt drivers to change to reverse gear. If the accelerator and brake are controlled very good, the parallel and vertical parking will be achieved easily with the help of automatic parking system. In 2013, Ford posted a system that achieved the real "automatic", it didn't need drivers to control the speed, braking, etc [2]. In 2015, the automatic parking system of Cruze's DCG flagship version came out, this system can achieve the automatic vertical and parallel parking completely based on infrared and ultrasonic radar sensors [3].

\section{Sensor Information Processing for Automatic Parking Environment Perception}

\subsection{Laser Data Pre-Processing}

There are some flawed sensor data because of the sensor's precision and environmental disturbances. That is, errors about ambient information that affect the accuracy of path planning caused by the mismatch of data acquired by sensor and the actual environment. In order to improve accuracy of environment recognition, for most sensor data, data preprocessing is required before data analysis. The preprocessing by using the least square method and the median filtering algorithm is applied in this paper.

Median Filtering Algorithm: The main idea of Median filtering is to is to run through the data in a digital image entry by entry, finding the median of neighboring entries and replacing each entry with it. The data points of a digital image or a set of data were sorted and replaced according to this principle.

Least Square Method: The Least Square Method is a standard approach in regression analysis. Setting $\left(\mathrm{x}_{\mathrm{i}}, \mathrm{y}_{\mathrm{i}}\right)$ $(\mathrm{i}=0,1, \cdots, \mathrm{m})$ for points of a dataset and $\mathrm{w}_{\mathrm{i}}>0(\mathrm{i}=0,1, \cdots, \mathrm{m})$ for the weight coefficient of each point. In function space $S=\operatorname{span}\left\{\varphi_{0}(\mathrm{x}), \varphi_{1}(\mathrm{x}), \cdots, \varphi_{\mathrm{n}}(\mathrm{x})\right\}$, and ask for $\mathrm{S}^{*}(\mathrm{x})$ in the Formula (1)satisfy the Formula (2).

$$
\begin{gathered}
s^{*}(x)=\sum_{k=0}^{n} \alpha_{k}^{*} \varphi_{k}(x) \in S \\
\sum_{i=0}^{m} \omega_{i}\left(s^{*}\left(x_{i}\right)-y^{i}\right)^{2}=\min _{S(x) \in S} \sum_{i=0}^{m} \omega_{i}\left(S\left(x_{i}\right)-y^{i}\right)^{2}
\end{gathered}
$$

The method of acquiring function $S^{*}(x)$ is called least square method of data fitting.

As we can see from Figure 1, median filtering can filter a small amount of noise data (as shown in Figure 1(b)), and the data smoothing could be much better by using the least square method together.

\subsection{Parking Space Detection Based on k-Means Algorithm}

The basic principle of K-Means clustering is elaborated as [4]: Firstly, k initial "means” are randomly generated within the data domain, Then, $\mathrm{k}$ clusters are created by associating every observation with the nearest mean. Finally, the centroid of each of the $\mathrm{k}$ clusters becomes the new mean. For example, the dataset is set as $\left\{\left[x_{i}\right], i=1,2, \cdots, n\right\}$.

Step 1: K1 [Initialization]: $k$ data points $\left\{c_{1}, c_{2}, \ldots, c_{k}\right\}$ of dataset $\left\{\left[x_{i}\right], i=1,2, \cdots, n\right\}$ are selected as the initial cluster centers.

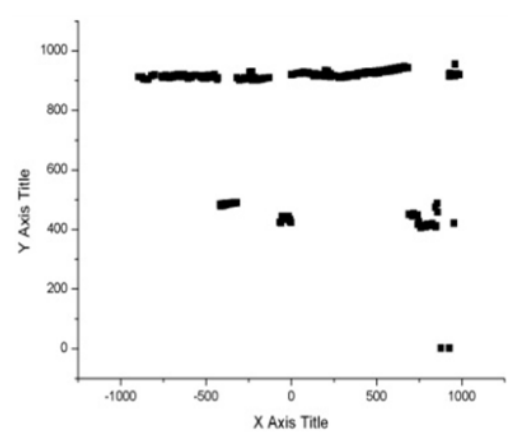

(a)

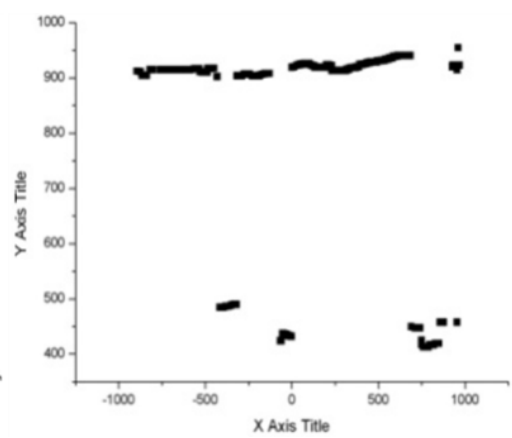

(b)

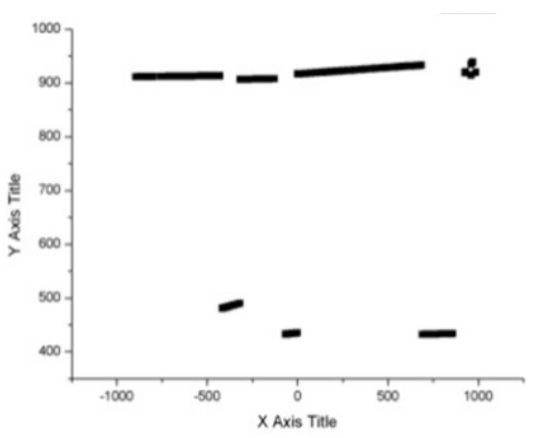

(c)

Figure 1. Contrast figure of data processing. (a) Raw laser data; (b) Processed by median filtering; (c) Processed by least square method. 
Step 2: K2 [Assign $x_{i}$ ]: Calculating the distance $d_{i j}, j=1,2, \ldots, k$ from $\left\{\left[x_{i}\right], i=1,2, \cdots, n\right\}$ to each initial cluster center $\left\{c_{1}, c_{2}, \ldots, c_{k}\right\}$, the data $x_{i}$ are classified into the nearest class.

Step 3: K3 [Revise $\left.c_{i}\right]$ : The new class cluster center of each class is acquired through calculating the average of all the data in each class.

Step 4: K4 [Deviation calculation]: $\mathrm{D}=\sum_{i=1}^{n}\left[\min _{r=1,2, \ldots, k} d\left(x_{r}, c_{r}\right)^{2}\right.$ ]

Step 5: K5 [Whether the $\mathrm{D}$ is convergent]: If $\mathrm{D}$ is convergent, the whole algorithm will end. Otherwise, it returns to $\mathrm{K} 2$ until convergence has been reached.

The parking space detection result is shown in Figure 2.

\section{Fuzzy Controller Design for Automatic Parking System}

\subsection{Establishment of Vehicle Kinematic Model}

The environment perception coordinate system of automatic parking system includes the global coordinate and the vehicle coordinate system. The origin of vehicle coordinate system is set to the midpoint of the rear axle. $\mathrm{X}$-axis is projected to the vehicle rear axle and directs right, and $\mathrm{Y}$-axis lies in the vehicle longitudinal axis and directs forward. The midpoint of the rear axle serves as the coordinates of the vehicle position in the global coordinate system. The course angle of the vehicle is shown as the direction of the vehicle and donated by $(x, y, \theta)$. Course angle is defined as the intersection angle between the vehicle longitudinal axis and the global $\mathrm{X}$-axis, and the positive direction is counterclockwise, which is shown in Figure 3.

$$
\left\{\begin{array}{l}
\dot{x}_{r}=-v \cdot \cos \theta \cos \varphi \\
\dot{y}_{r}=-v \cdot \sin \theta \cos \varphi \\
\dot{\theta}=v \cdot \frac{\sin \varphi}{L}
\end{array}\right.
$$

Regarding the vehicle as a rigid body with low speed and assuming vehicle movement direction is consistent with the movement direction of rear wheels, and the motion trajectory of rear wheels could be considered as the motion trajectory of the vehicle body. Under this condition, the center coordinate of the rear wheel axis $\left(x_{r}, y_{r}\right)$

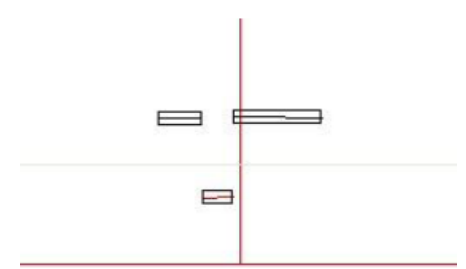

(a)

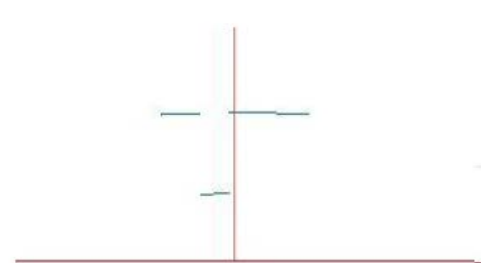

(b)

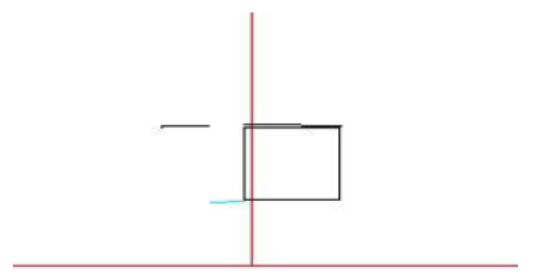

(c)

Figure 2. Parking space detection based on K-means clustering method. (a) Pre-processed laser data; (b) Obstacle detection result; (c) Parking space detection.

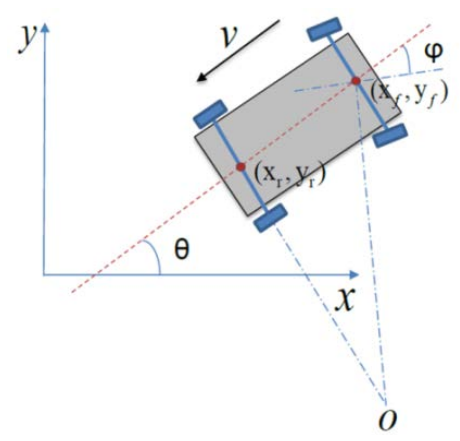

Figure 3. The vehicle coordinate system. 
can be considered as vehicle body's coordinate and the trajectory of the vehicle can be expressed by $\left(x_{r}, y_{r}, \theta\right)$.

\subsection{The Fuzzy Control Simulation of Vertical Parking System}

The membership function of each input and output and the parameter settings of fuzzy controller is elaborated in literature [5]. The driver needs to stop the vehicle initially before starting the automatic parking system. But it is very difficult for most people to make the vehicle body parallel to the lane direction perfectly at initial point. Even if the body course angle is zero, the initial course angle $\theta$ of body should be controlled between $-10^{\circ}$ $10^{\circ}$. Three different kinds of simulation results are shown below when the initial course angle $\theta$ is $-10^{\circ}, 0^{\circ}$ and $10^{\circ}$ respectively.

Red rectangle represents the car model in Figure 4 and the black thick solid line represents the border of the parking space. It is said in Figure 4 that the vehicle won't encounter the border in the process of reversing. The course angle of the vehicle body is $90^{\circ}$ at the end of automatic parking. The change curve of the vehicle course angle is shown in Figure 5.

If $\theta=10^{\circ}$, movement track of vehicle and the change curve of the vehicle course angle are shown in Figure 6 and Figure 7.

When the initial course angle is $10^{\circ}$, it is necessary for successfully parking to keep unchanged course angle for a period of time, then gradually increase its value when the vehicle close enough to the parking space, as it is shown in Figure 6. The vehicle has been parked successfully as shown in two figures below and the eventually course angle is $90^{\circ}$.

If $\theta=-10^{\circ}$, movement track of vehicle and the change curve of the vehicle course angle are shown in Figure

\section{8 and Figure 9.}

When the initial course angle $\theta=-10^{\circ}$, the course angle should be increased obviously to make the automobile aligned quickly. The simulation results are shown in Figure 8 and Figure 9.

Compared three different kinds of parking simulation results. We can draw the conclusion that the parking simulation is best when $\theta=0^{\circ}$. When $\theta$ is $-10^{\circ}$ or $10^{\circ}$, the parking simulation result is not as better as $\theta=0^{\circ}$.

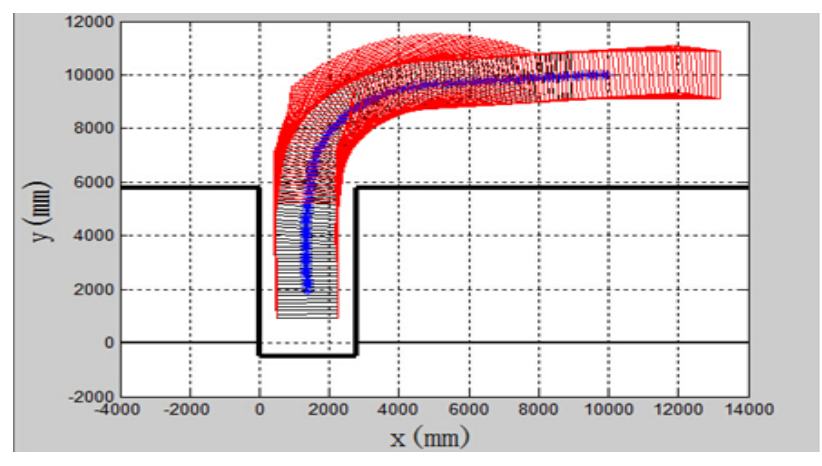

Figure 4. $\theta=0^{\circ}$, movement track of vehicle when vertical parking.

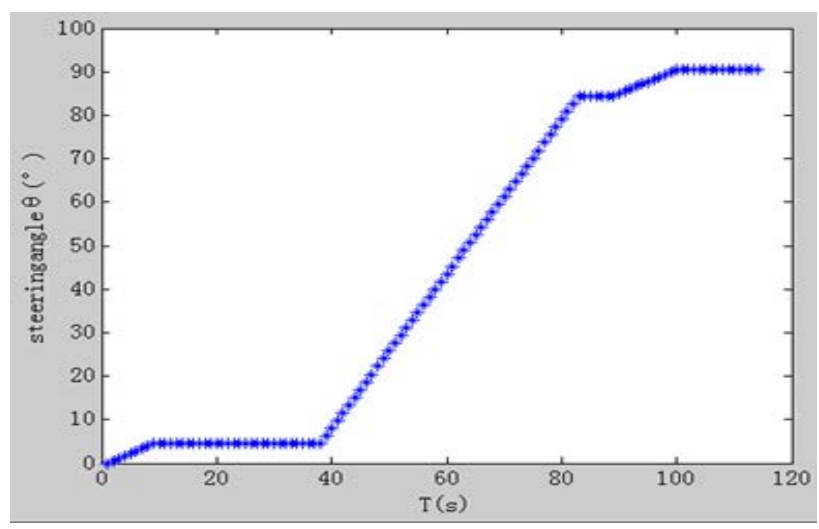

Figure 5. $\theta=0^{\circ}$, the change curve of the vehicle course angle. 


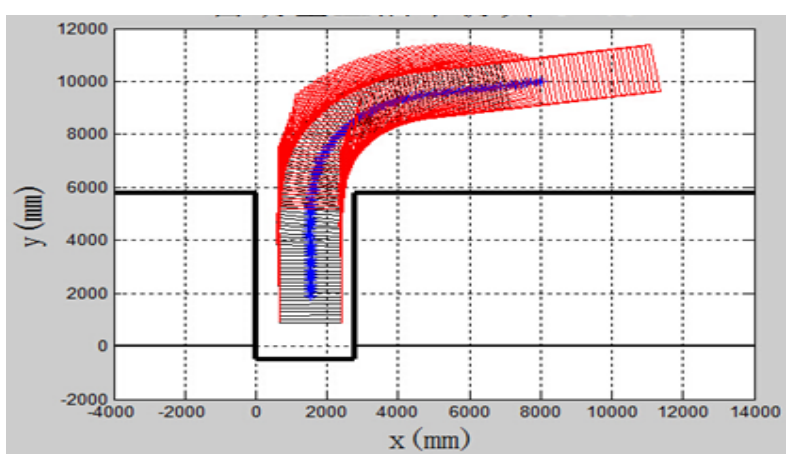

Figure 6. $\theta=10^{\circ}$, movement track of vehicle when vertical parking.

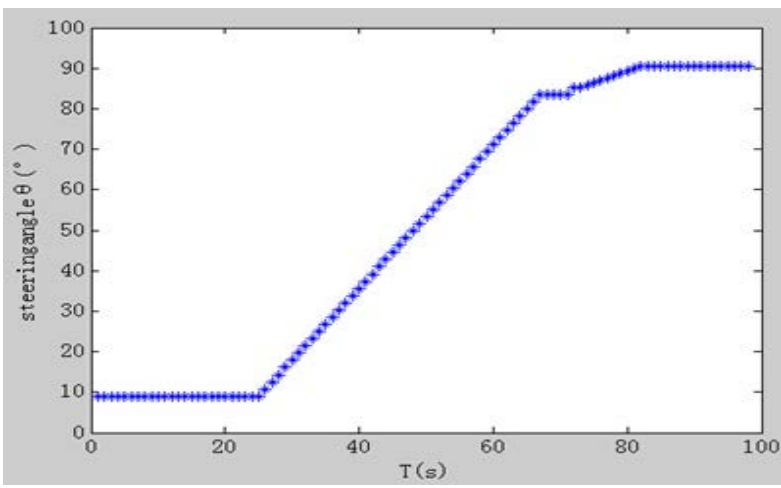

Figure 7. $\theta=10^{\circ}$, the change curve of the vehicle course angle.

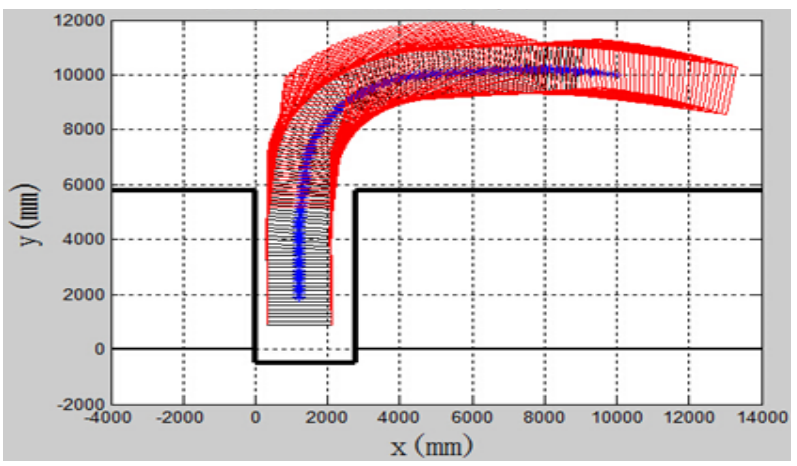

Figure 8. $\theta=-10^{\circ}$, movement track of vehicle when vertical parking.

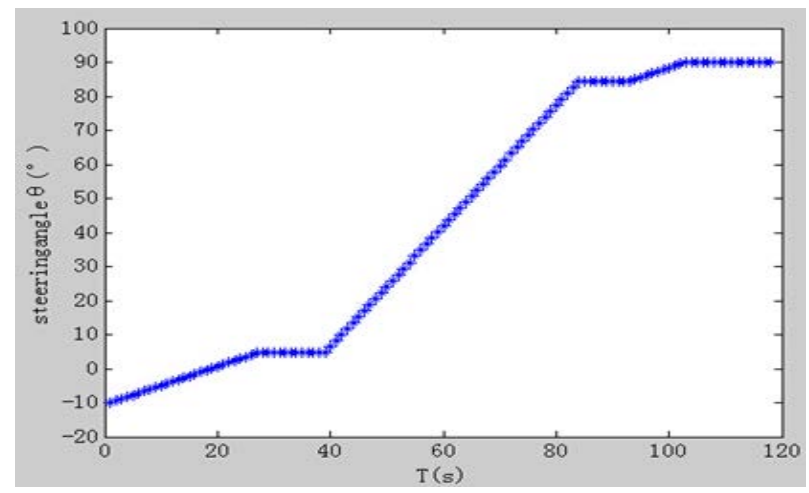

Figure 9. $\theta=-10^{\circ}$, the change curve of the vehicle course angle. 


\section{Conclusions}

Next key point is to establish the vehicle kinematic model and design the fuzzy controller for automatic parking system, then carry out simulation on both the automatic vertical parking and parallel parking process. This section firstly established automobile kinematic model by using Arman kinematic model and simplified the vehicle dynamics model during the parking process. The second step is to gain the appropriate membership functions and fuzzy rules in fuzzy toolbox of Matlab. Setting the coordinate of the vehicle (x, y, $\theta$ ) as the inputs and the front wheel steering angle as the output of the fuzzy controller. The simulation results showed the perfect feasibility and real-time.

Since the parking simulation results are acquired based on the assumption of low speed station, the Arman vehicle dynamics model is employed in actual situation instead of the kinematic model. So improving the model and carrying out related research sunder other speed conditions need further works.

\section{References}

[1] Shao, B. (2013) Research of Environment Perception for Automatic Parking System Based on Laser Scanner. Dalian University of Technology.

[2] http://www.chexun.com/2013-04-21/101827358.html

[3] http://v.bitauto.com/vplay/345284.html

[4] Na, S., Liu, X.M. and Yong, G. (2010) Research on k-Means Clustering Algorithm: An Improved k-Means Clustering Algorithm. 2010 Third International Symposium on Intelligent Information Technology and Security Informatics (IITSI), Jinggangshan, 2-4 April 2010, 63-67. http://dx.doi.org/10.1109/IITSI.2010.74

[5] Wang, H. (2014) The Research of Environment Perception Technology for Automatic Parking Based on Laser Scanner, The Master Degree Dissertation, Dalian University of Technology, Dalian. 